\title{
Manifestations and reduction strategies of stigma and discrimination on people living with HIVIAIDS in Tanzania:
}

\author{
P. MUTALEMWA*, W. KISOKA, V. NYIGO, V. BARONGO, M.N. MALECELA \\ and W.N. KISINZA, \\ National Institute for Medical Research, P O Box 9653, Dar es Salaam, Tanzania
}

\begin{abstract}
HIV/AIDS-related stigma, and its associated discrimination, is known to negatively affect all aspects of HIV prevention, care and treatment. Studies have revealed the extent to which individuals are stigmatized and discriminated against the health care system. However, there has been limited information on stigma manifestations and reduction interventions. The main objective of the study was to determine the magnitude and factors influencing HIV/AIDS-related stigma and discrimination with a specific focus on the manifestations and reduction interventions. In-depth interviews, exit interviews and focus group discussions were deployed in the study. Results have shown that HIV/AIDS is increasingly conceptualized as a continuum between prevention and care, effects of stigma and discrimination are from both health facilities and communities. While religious leaders isolate people living with HIV/AIDS (PLWHAs) and consider them as most promiscuous, health workers also have strong negative attitudes and feelings and as a result PLWHAs refrain from counselling and testing services. A stigmatizing social environment was found to pose barriers to all strategies and/or interventions that are aimed at reducing this situation. Because of stigma and discrimination, people living with HIV/AIDS receive inadequate treatment, as such they decline to divulge their status to partners or change their behaviour avoiding depressing reactions. Basing on that therefore, adequate outreach services at both community and health facility levels be established and line up in the fight against stigma and discrimination facing people living with HIV/AIDS.
\end{abstract}

Key words: Stigma, discrimination, manifestation, interventions, HIV/AIDS, Tanzania

\section{Introduction}

Stigma refers to a set of attitudes and beliefs, at individual and societal levels, that are based on contemporary social norms and values, and which result in discrimination, marginalization, and outright prejudice against individuals and groups in a given society (Sayce, 1998). A stigmatized person is someone who possesses an undesired difference or deviance, while discrimination is any form of distinction, exclusion, or restriction affecting a person because of a personal characteristic. Research shows that discrimination is most frequently associated with diseases that have severe outcomes and whose modes of transmission are perceived to be under a person's control, criteria that fit HIV/AIDS. Through stigma, society often blames infected people for being ill and asserts the innocence and health of those who stigmatize (Link et al., 1997).

Stigma exists when the following interrelated components converge. In the first component, people distinguish and label human differences. In the second, dominant cultural beliefs link labelled persons to undesirable characteristics to negative stereotypes. In the third, labelled persons are placed in distinct categories so as to accomplish some degree of separation of "us" from "them." In the fourth, labeled persons experience status loss and discrimination that lead to unequal outcomes. Stigmatization is entirely contingent on access to social, economic and political power that allows the identification of differences, the construction of stereotypes, the separation of labelled persons into distinct categories and the full execution of disapproval, rejection, exclusion and discrimination. Thus the term stigma is applied when elements of labelling, stereotyping, separation, status loss and discrimination co-occur in a power situation that allows them to unfold (Devine et al., 1999).

Stigma is a common human reaction to disease and throughout history many diseases have

* Correspondence: Nkechi G. Onyeneho; E-mail: nkechux@yahoo.com 
carried considerable stigma, these include leprosy, tuberculosis, cancer, mental illness and a number of sexually transmitted diseases (STDs). HIV/AIDS is the latest disease to be stigmatized. Pervasive stigma has surrounded HIV/AIDS since the beginning of the pandemic and it has mostly been accompanied by discrimination, affecting transmission patterns and access to care, treatment and support. It negatively affects preventive behaviours such as condom use, HIV test-seeking and care-seeking behaviour upon diagnosis as well as quality of care given to People Living with HIV/AIDS (PLHA). One of its most surprising elements of is its ever present nature even where the epidemic is widespread and affecting so many people, such as in sub-Saharan Africa (UNAIDS 2000). Despite of the perceived knowledge on disease transmission, the epidemic has been accompanied by fear, ignorance, and denial, leading to repeated incidences of stigmatization and discrimination against PLHA and in some cases their families (Mann, 1987; Herek \& Glunt, 1988).

Tanzania, like other countries in Sub-Saharan Africa, stigma against HIV/AIDS remains very strong and plays a major role in fuelling HIV infection. In health care settings, stigma is the result of inability of health workers to understand and manage HIV/AIDS. They see no remedy or solution to the disease and feel that, they do not have the psychological resources to cope with somebody whose death is inevitable (Kisinza et al., 2002). Health care workers fulfil societal roles that place them in uniquely important positions to prop up or dissolve AIDS-related stigma. They have a special obligation to protect and assist people living with HIV/AIDS through public health education. However, they may accept and promote HIV/AIDS-related stigma, either consciously or unconsciously. Health care workers have inadequate access to universal precautions, such as gloves, post-exposure prophylaxis and safe blood collection kits. Because of inadequate protective measures, health care workers' fears of contagion are fuelled by "unsafe" contact with positive patients (Kisinza et al., 2002).

Although stigma has widespread impact on HIV/AIDS prevention programmes, there is lack of data to inform the designers of interventions to reduce stigma and discriminatory practices. This study was therefore carried out to determine the magnitude and factors influencing HIV/AIDS related stigma and discrimination in Tanzania.

\section{Materials and Methods}

\section{Study area and data colletion}

A total of 18 sites in nine regions of Tanzania were involved in the study. The sites were selected basing on the high HIV prevalence in accordance to the data available from the respective regions (TDHS, 2005).The study involved Morogoro urban, Kilombero, Dodoma, Mpwapwa, Mtwara, Masasi, Tanga city, Muheza, Mbeya City, Kyela, Mwanza city, Geita, Kinondoni, Temeke, Iringa, Mufindi, Kigoma and Kasulu.

Qualitative methods of data collection were used to gather information regarding HIV/AIDS stigma and discrimination. The methods used were in-depth interviews, exit interviews and focus group discussions. In-depth interviews were conducted to eight health workers in each district and involved District AIDS co-ordinators, PMTCT counsellors, In-charge of HIV/AIDS units, Maternal and Child Health Coordinators, District Nursing Officers, Doctor in-charge of the Regional and District hospitals, and In-charge of labour wards. In-depth interviews were also done to 36 religious leaders from Christian and Muslin denominations and 18 traditional practitioners. Exit Interviews were conducted to PLHAs mainly those taking the ARVs at the health facilities. Two focus group discussions involving combined groups of males and youth and male and females were also conducted in each district. Additionally several communities were also visited in order to get their knowledge and perceptions.

\section{Data analysis}

The taped in-depth interviews and focus group discussions were transcribed. The transcriptions were then analysed using the constant comparative method. Through open coding, common themes of what emerged from the collected data were developed and examined in relation to their context, meanings, and circumstances. Similarities and differences about the compiled codes were clustered together to 
create categories. In order to have a clear analysis, conceptual saturation was reached when no new categories were generated.

\section{Results}

Respondents in the in-depth interviews and focus groups discussions had a wide-ranging understanding of stigma and discrimination. However, the general definition captured was pointed out as being a state whereby people portray verbally or bodily negative attitudes towards a person or a group of people due to their particular characteristics or physical attributes. They defined discrimination as a state of deliberately isolating a person or a group of people from participating in various socio-economic and political aspects of life due to certain attributes. According to respondents' point of view, there are two types of stigma; firstly self stigma (fear) where a person reduces himself/herself from a whole and usual person to a tainted and discounted one. Secondly stigma from others where people show negative attitudes towards a person or a group of people due to their particular status. "When a person knows his/her HIV status, helshe becomes fearful and begins to stigmatize him/herself with uncomfortable feelings all the time thinking that life has reached to an end" (male, 54 years, Dodoma).

HIV/AIDS is the disease that threatens people because of its incapacitating nature. Because of that, there is also a misconception which is fuelled by the physical appearance of the affected. The stage of the disease a patient reaches is what determines the level of stigma and discrimination he/she will face. From interviews and focus group discussions, respondents associated HIV transmission with sexual intercourse, and that the disease was mainly connected to promiscuity.

Respondents unanimously cited poverty as being one of the major causes of stigma and discrimination against PLWHAs. People living with HIV/AIDS tended to be abandoned by relatives and friends once it is known that they are infected. They were seen as a burden and as such responsible for their deeds and that they should pay for their sins. On the other hand, respondents claimed that HIVIAIDS was often affecting poor than the rich people. Respondents were of the opinion that despite the fact that isolation of HIV services from other services was meant to bring privacy to those infected and to ensure maximum attendance of them, it portrays stigma to those infected and those wishing to seek services for fear of being recognised by people who know them.

Lack of proper training and protective gears for HIV/AIDS case management was also said to be another factor causing stigma. Health personnel were concerned with lack of proper training on how to manage AIDS patients among community members. On the other hand community members were afraid of attending AIDS patients due to fear of being infected when they do not have protective gears. According to respondents, HIV/AIDS related stigma and discrimination were demonstrated either verbally or by actions. The belief that one can get infected by touching an affected person caused people to keep distance with patients. Stigma and discrimination were further portrayed through isolation or abandonment of an AIDS patient within the household or outside. Within the household isolation was reported to restricting patients from sharing utensils during eating and sleeping. Moreover, people feared to manage HIV patients or those who die from AIDS. This was also perpetuated by the contradictory educational messages on televion, radio or other information, education and communication materials which indicate that one cannot get HIV through sharing of food or sleeping places.

At the community level, name calling, mocking and pointing fingers at those infected, abusive language to HIV patients, HIV patients' delay in and/or neglect of seeking services were among the most common manifestations of stigma and discrimination. Others were denial of training opportunities, appointment, promotion, and career advancement. Stigma and discrimination were also shown by health workers at health facilities. Health workers were said to use of abusive language, or may refuse or delay attending and managing HIV patients especially those admitted to healthcare facilities. In other circumstances respondents complained about over- billing of other medical services to HIV patients. Since the patients were getting the HIV service free of charges, health workers tended to 
overcharge them for other services.

Healthcare workers reported low acceptance of post-exposure prophylaxis (PEP) when they accidentally injure themselves during provision of health services to HIV/AIDS patients. Taking PEP is associated with stigma. This was because HIV/AIDS was associated with mal-behaviour such as promiscuity and drug use.

Most affected persons tended to be open only to people known to themselves or close to them. This was exemplied by statements from three interviewees: "After my HIV+ status was known, the people I was sharing a rented house started to discriminate me by not using the same toilet with me and to others moved out to another place" (Female-PLWHA, 35 years old, Kigoma). "People are afraid to go for HIV testing because they have witnessed those who are known to be HIV infected being mistreated. It is therefore, very difficult to go for HIV testing for fear of facing the same consequences" (Male, 45 years old, Mtwara). "When you go to the hospital a doctor tells you why you do bother us, don't you know what your problem is?" (Male, 41 years old, Kigoma).

According to respondents, reaction after confirming HIV+ took different forms. It may sometimes lead to depression, anxiety, fear, weeping and even loss of consciousness. It takes time before ones status is been accepted. Life becomes meaningless to the affected especially when they do not accept the reality. However, not all are bearing the same notion as respondents pointed out that with the availability of free ARV drugs; many have regained hope marking the progress of normal life routine. "When I firstly tested positive, I did not believe it, I went to the hospital more than three times, and results were the same. I felt really bad but now I have accepted my status" (Male-PLHA, 47 years old, Geita). Other PLHAs had these to say: "When I received my HIV results I felt very relieved, I knew it was beginning of a new life I told my relatives and friends about it and they did not believe" (Male, 29 years old, Iringa). "I took an HIV test when my health was okay, when I found that I was infected I did not believe it. I cried a lot and was so depressed for a long time until when I joined SHIDEPHA+ and realized that we are so many with this problem and felt comfortable" (Male-PLHA, 34 years old, Masasi.
During the exit interviews with people on ARVs, it was pointed out that willingness to disclose their HIV+ status to people was very subjective. There were those who said that they were willing to disclose their status because they expect assistance from relatives, friends and colleagues. However most of them would disclose their status to very close relatives. While men said they may disclose their status to wives and not otherwise. However, some women in discussion group discussants pointed out that they tend to keep their status unknown mainly for fear of consequences such as divorce. Youth were more willing to disclose to the third party mainly clinicians or counsellors or very close friend/ relative. Among the reasons that were mentioned for not disclosing their status were fear of the repercussions such as stigma and discrimination, loss of jobs, denial of education opportunities and even marriage prospects, disappointing or shocking their relatives, spouses or children. "If I am found to be HIV+I will only tell my mother because other people will degrade me" (Female, 21 years old, Tanga). "People mock and abuse you saying that is what you deserve (Female-PLHA, 39 years, Kigoma).

Interventions and strategies were explored from health care settings, religious and traditional practitioners and were as follows. Socio-cultural groups addressing effects of stigma and discrimination were seen as a major force towards educating people on the effects of stigma and discrimination. The Health care setting was mentioned as being the frontrunner in combating HIV/AIDS related stigma and discrimination. There were home visits by health care workers, outreach services, seminars with health workers, community based health education (on taking care of HIV/AIDS patients), training health workers on the effects of HIV/AIDS related stigma and discrimination, integration of HIV/AIDS testing services and outpatients, formation of AIDS committee at workplaces and other unique settings admission of AIDS patients with other patients in the same ward and use of protective gears among health care workers when attending HIV patients.

Religious institutions were mentioned as the most powerful venues to curb HIV/AIDS-related stigma and discrimination. It was pointed out by both community members and PLWHAs that 
religious leaders have been campaigning and mobilizing actions against stigma and discrimination. PLWHA groups were reported to have been supported financially with micro finances in order to raise their incomes. Religious leaders through preaching have via preaching have successfully mobilized community members as well as PLWHA into the fight against HIV/AIDS related stigma and discrimination. Traditional healers have been encouraging and nurturing PLWHA organizations, and were considered to be crucial in reducing stigma and discrimination. When interviewed traditional healers pointed out that strengthening health education has been very important in building alliances between PLWHAs and other segments of communities and this in its own has reduced HIV/AIDS related stigma and discrimination.

\section{Discussion}

The findings from this study demonstrate that stigma and discrimination relating to HIV/AIDS is still a big problem and undermines public health efforts to combat the epidemic. HIV/AIDS related stigma negatively affects preventive behaviours such as condom use, HIV test-seeking behaviour, and care-seeking behaviour upon diagnosis, quality of care given to HIV-positive patients, and perception and treatment of PLWHAs by communities and families. HIV/AIDS related stigma is common even where the epidemic is widespread and affecting so many people (Fortenberry, 2004). HIV/AIDS-related stigma is one of the key obstacles in accessing care for people and families affected by this pandemic. Many people who are infected do not know their status either due to lack of facilities, lack of appropriate information or due to fear of stigma. Stigma is preventing people from seeking early care for tuberculosis and other opportunistic infections, seeking counselling and testing services, accessing and receiving good quality care and also preventing people from adhering to treatments where they are available such as mother-to-child transmission. An effort to reduce such stigma is therefore, a vital pre-requisite in both stemming the pandemic and providing adequate care and support to those affected.

In health care settings, stigma is the result of inability of health workers to understand and manage HIV/AIDS. They see no remedy or solution to the disease and feel that, they do not have the psychological resources to cope with somebody whose death is inevitable. As individuals they have an essential role to play in educating the public about HIV/AIDS. Indeed, health care workers' tendencies to engender and promote AIDS-related stigma is widespread throughout the African continent and across the world (Mahendra et al., 2007).

In Tanzania, despite heavy investments in campaigns and education aimed at reducing the transmission rate, the disease has continued to increase, affecting mostly the youth who are at the productive age. Because a cure and vaccine for HIV/AIDS is not expected in the near future, its prevention is the only means of reducing the spread of the disease. Thus, as has been true for most major communicable diseases, public health measures are likely to have a greater cumulative impact than medical ones. In the case of HIV/AIDS, knowledge about the transmission of the disease and subsequent change of high-risk behaviours are presently the best weapons the public health community has. While it may be unrealistic to think that we can eliminate stigma and discrimination altogether, this study has shown that something can be done through a variety of interventions including information, counselling, coping skills acquisition and contact.

HIV-related stigma and discrimination remains an enormous barrier to effectively fighting the HIV and AIDS epidemic. However, in this study it was pointed out by many respondents that fear of discrimination often prevents people from seeking treatment for AIDS or from admitting their HIV status publicly. This is because people with or suspected of having HIV may be turned away from healthcare services or employment. In some cases, they may be evicted from home by their families and rejected by their friends and $c$ olleagues. The stigma attached to HIV/AIDS can extend into the next generation, placing an emotional burden on those left behind. Combating the stigma and discrimination against people who are affected by HIV/AIDS is as important as developing interventions that address and hence reduce HIV/AIDS related stigma and discrimination. 
The religious sector has the potential roles in providing care, comfort, and spiritual support to HIV-infected and -affected communities. The religious sector is expected to play a lively role not only promoting preventive messages about HIV/AIDS but also leading the fight against stigma in diverse settings. However, the sector has not yet comprehensively addressed the ambivalent and often negative interpretation of sexuality that is common in church dogma, and this ambivalence has been transformed into widespread fear of people living with HIV and cruelty towards them. The origins of stigma in the religious sector are based upon stringent concepts of sexuality and morality. The reduction of stigma can only occur if religious leaders make conscious efforts to change their own behaviour and promote integrated care programmes for people living with HIV/AIDS.

\section{Acknowledgements}

The authors are grateful to the study participants for their willingness to participate in the study. They also wish to extend their sincere thanks to the respective Regional and District Medical Officers for their mutual support during the study. Thanks to the National Institute for Medical Research for the invaluable support that enabled a smooth undertaking of this survey. The study received financial support from the President's Emergency Plan for AIDS Relief (PEPFAR) through CDC/NIMR Co-Agreement on HIV/AIDS care and treatment (No. U62CCUO24211-01).

Received 4 June 2008

Revised 19 June 2008

Accepted 21 June 2008

\section{References}

Devine, PG, Plant,E. A, Harrison K.(1999) “The problem of "us" versus "them" and AIDS stigma." American Behavioural Scientist 42:1212-1228.
Fortenberry, D.J. (2004) The Effects of Stigma on Genital Herpes Care-seeking behaviours: Mid-America Adolescent STD Cooperative Research Center, Department of Pediatrics, Indiana University School of Medicine, Indianapolis, USA.

Herek, G \& Glunt, E.K. (1998) An epidemic of stigma: Public reaction to AIDS. American Psychologist 43(11): 886-891

Kisinza, W., Makundi E., Mwisongo, A., Mubyazi G., Magesa, S., Malebo H., Mcharo J., Senkoro, K., Hiza P., Pallangyo, K., Ipuge, Y., Kitua, A. \& Malecela-Lazaro, M. (2002) Stigma and discrimination on HIV /AIDS in Tanzania. Tanzania Health Research Bulletin 4, 42-46.

Link, B.G., Struening, E.L., Rahav, M., Phelan, J.C. \& Nuttbrock, L. (1997) On stigma and its consequences: evidence from a longitudinal study of men with dual diagnoses of mental illness and substance abuse. Journal of Health and Social Behaviour 38, 177-190.

Garbus, L. (2004) HIVIAIDS in Tanzania. AIDS Policy Research Centre, University of California San Francisco, 93pp.

Mahendra, V.S., Gilborn, L., Bharat, S., Mudoi, R., Gupta, I., George, B., Samson, L., Daly, C. \& Pulerwitz, I. (2007) Understanding and measuring AIDS-related stigma in health care settings: A developing country perspective. Journal of Social Aspects of HIVIAIDS, 4, 616-625.

Mann, J. (1987) Statement at an informal briefing on AIDS. The $42^{\text {nd }}$ Session of the United Nations General Assembly, 20 $0^{\text {th }}$ October New York

Sayce, L. (1998) Stigma, discrimination and social exclusion: what's in a word?" Journal of Mental Health, 7:331-343.

TDHS (2005) ) Tanzania Demographic and Health Survey 2004-2005. United Republic of Tanzania.

UNAIDS (2000) Protocol for the identification of discrimination against people living With HIVIAIDS: Best Practice Collection. United Nations Programme on HIV/AIDS. Geneva. 